\title{
The Effects of Different Congestion Management Algorithms over Voip Performance
}

\author{
Szabolcs Szilágyi \\ Faculty of Informatics \\ University of Debrecen \\ Debrecen, Hungary
}

\begin{abstract}
This paper presents one of the features of DS (Differentiated Services) architecture, namely the queuing or congestion management. Packets can be placed into separate buffer queues, on the basis of the DS value. Several forwarding policies can be used to favor high priority packets in different ways. The major reason for queuing is that the router must hold the packet in its memory while the outgoing interface is busy with sending another packet. The main goal is to compare the performance of the following queuing mechanisms using a laboratory environment: FIFO (First-In First-Out), CQ (Custom Queuing), PQ (Priority Queuing), WFQ (Weighted Fair Queuing), CBWFQ (Class Based Weighted Fair Queuing) and LLQ (Low Latency Queuing). The research is empirical and qualitative, the results are useful both in infocommunication and in education.
\end{abstract}

Keywords-CBWFQ; congestion; CQ; FIFO; LLQ; Pagent; $P Q$; queuing; WFQ

\section{INTRODUCTION}

At the beginning computer networks were designed mainly for data transfer such as FTP and email, where delay was considered to be unimportant. In most cases the delivery service was effective, and the TCP protocol dealt with data losses. As the multimedia applications became popular (voice transfer, video conferences), separate telephone and video communication networks were set up (see Fig. 1). Nowadays, office and company networks are transformed into one converged network (see Fig. 2), in which the same network infrastructure is used to ensure all the requested services [1].

Although converged networks have many advantages, there are some disadvantages too, namely the competition for network resources (buffers of routers), which leads to congestion. Delay in delivering the packets, jitter, loss of packets are consequences of congestion. Different applications show different sensitivity to these issues. For example, FTP is not impacted by delay and jitter, whereas the multimedia applications (e.g. interactive voice) are very sensitive to them and the loss of packets too [2]. Quality of Service (QoS) was introduced to handle this problem, and it is able to provide different priority to different applications, users, or data flows, or to guarantee a certain level of performance to a data flow [3], [4].

This research was realized in the frames of TÁMOP 4.2.4. A/2-11-12012-0001 „National Excellence Program - Elaborating and operating an inland student and researcher personal support system". The project was subsidized by the European Union and co-financed by the European Social Fund.

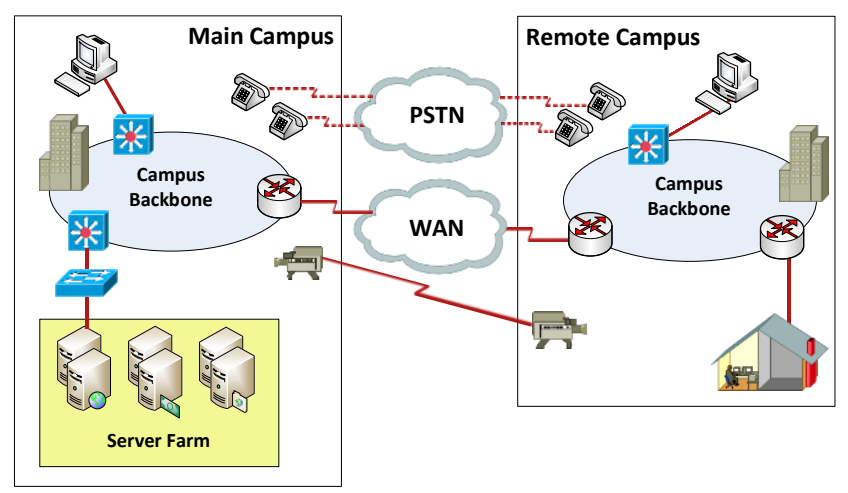

Fig. 1. A classical non-converged network

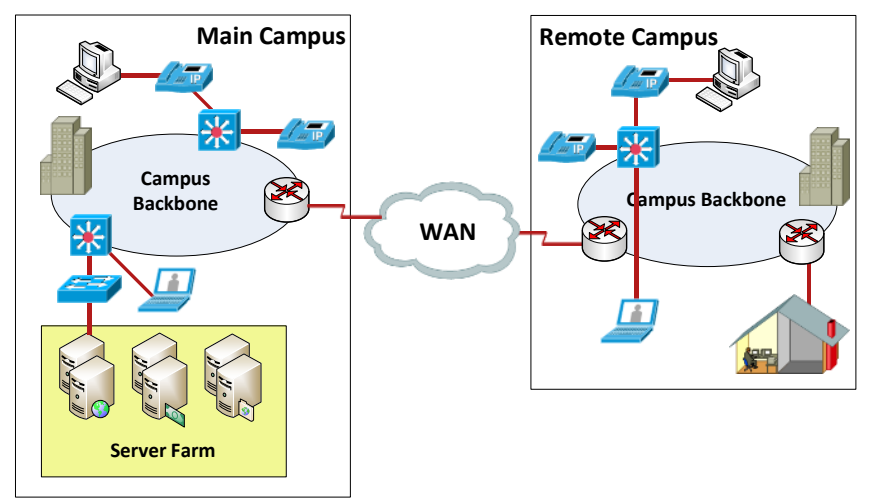

Fig. 2. A converged infocommunication network

In accordance with the QoS requirements and recommendations for the interactive voice traffic packet loss should be no more than $1 \%$, one-way latency should be not exceed $150 \mathrm{~ms}$ and the average one-way jitter should be targeted at less than $30 \mathrm{~ms}$ [5].

In the IP header there are some fields which can be used to make distinction between the packets of different applications, for example the Type of Service (ToS) field [6]. Different technics are used for congestion management (PQ, CQ, WFQ, CBWFQ and LLQ). Congestion avoidance (WRED), traffic shaping and traffic policing are also used by the QoS technology in order to control data traffic [7]. This article focuses on the most important component, namely the congestion management.

Our purpose is to analyze and evaluate the efficiency of these congestion management algorithms using a laboratory 
environment. This paper examines the following methods: FIFO, PQ, CQ, CBWFQ, WFQ and LLQ. It is important to note that these algorithms have real effect only in the case of congestion.

The network topology for the performance evaluation is identical to the one used in former articles (see e.g. [8]-[10]). This paper continues to study the queuing technologies for congestion management. In [8] and [11] the authors considered three algorithms: FIFO, PQ and WFQ. The conclusion was that WFQ is the most efficient for multimedia applications. In addition to these three new algorithms were investigated: CQ, CBWFQ and LLQ. The main result of this paper is that for multimedia applications (mainly for voice transfer) LLQ is more efficient then WFQ.

The detailed description of the algorithms has been discussed in several papers already (see e.g. [12]-[14]).

\section{THE MEASUREMENT ENVIRONMENT}

The measurement environment network topology is shown on Fig. 3, which was built at the network laboratory of the Faculty of Informatics, University of Debrecen.

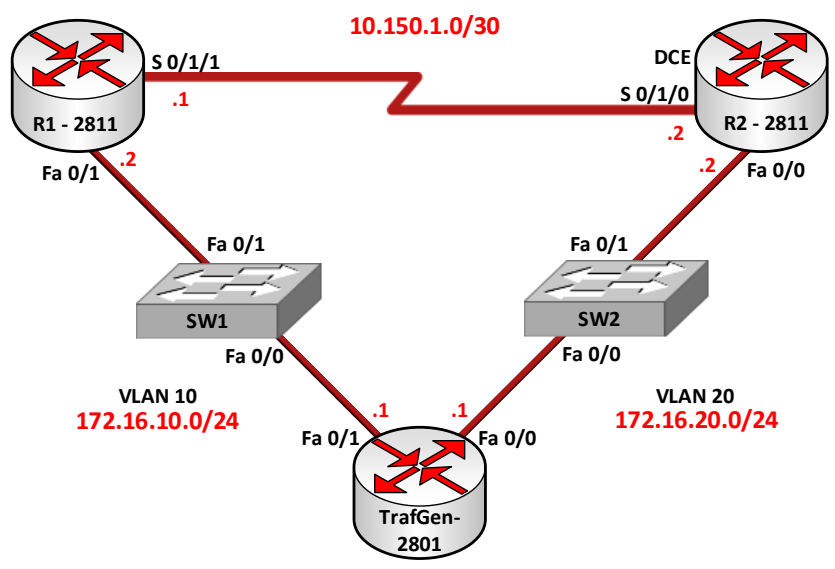

Fig. 3. The measurement environment

The measurement environment consists of three routers (two Cisco 2811 type routers and one Cisco 2801 router) and two switches. The routers are connected with a point-to-point link, having the speed of 128000 cycles per second. The rest of the network devices are connected with $10 \mathrm{Mbps}$ Ethernet links. The part between the two routers is actually a narrow cross-section where congestion can happen. For this reason the congestion management algorithms are activated in this area (see [15]-[16]).

The Cisco IOS 12.4 operating system was running on the $R 1$ and $R 2$ routers, represented on Fig. 3. The TrafGen router was responsible for the functioning of the communication endpoints. This was used to generate the traffic, and the operating system run on TrafGen was c2801-tpgen+ipbasemz.PAGENT.4.3.0 [17], which enabled the traffic generation, attached timestamps to the outgoing packages, and performed the statistical analysis based on the rate of incoming packets.

In order to distinguish between the generated and incoming traffic, two Cisco 2960 switches were used (SW1, SW2). These created two Virtual LANs, namely VLAN 10 and VLAN 20 [18]. A serial connection was created between $R 1$ and $R 2$, which simulated a slow WAN. Three types of traffic were generated, similarly to the previous papers: an FTP, Video and VoIP traffics. In the next section the traffic generation code is shown used by TrafGen router.

\section{A. The traffic generation}

The following code was used for traffic generation [19]:

wait-after-stop 1 ! Waiting time (sec)

!FTP traffic generation

fastethernet0/1 ! The TrafGen router output interface name

add tcp ! Adding a traffic stream (TCP)

datalink ios-dependent fastetherneto/1 !

The output interface name

12-arp-for 172.16.10.2 ! Layer 2 ARP message to default gateway

13-src 172.16.10.1 ! Layer 3 source IP address

13-dest 172.16 .20 .1 ! Layer 3 destination IP address

13-tos 0x00 ! Layer 3 packet header ToS byte value

14-src 21 ! Transport layer source port number

14-dest 21 ! Transport layer destination port number

name FTP ! Name of the generated traffic

rate 20 ! Setting the packet send rate

length 1434 ! Setting packet length (Bytes)

delayed-start 0 ! Delaying start of packet generation

(sec)

send 206 ! Sending packets

fastethernet0/0 ios-dependent capture

! The TrafGen router input interface name

!VIDEO traffic generation

fastethernet $0 / 1$

add tcp

datalink ios-dependent fastethernet0/1

12-arp-for 172.16.10.2

$13-\operatorname{src} 172.16 .10 .1$

13-dest 172.16 .20 .1

$13-\operatorname{tos} 0 \times 22$

$14-\operatorname{src} 4249$

14-dest 1720

name VIDEO

rate 50

length 1500

burst on ! Sending traffic stream in bursts

burst duration off 1000 to 2000

burst duration on 1000 to 3000

delayed-start 0

send 333

fastethernet0/0 ios-dependent capture !VOICE traffic generation

fastethernet $0 / 1$

add udp

datalink ios-dependent fastethernet0/1

12-arp-for 172.16.10.2

$13-\operatorname{src} 172.16 .10 .1$

13-dest 172.16 .20 .1 
13-tos $0 \times 2 \mathrm{E}$
14-src 44899
14-dest 5060
name VOICE
rate 50
length 150
delayed-start 0
send 513
fastethernet0/0 ios-dependent capture

\section{B. The implementation of congestion management algorithms}

The part between the $\mathrm{R} 1$ and $\mathrm{R} 2$ routers is actually a narrow cross-section where congestion can happen. For this reason the congestion management algorithms were activated in this area (between the $R l^{\prime} \mathrm{S}^{\mathrm{S}} 0 / 1 / 1$ and $R 2^{\prime}{ }^{\prime} \mathrm{S} 0 / 1 / 0$ interfaces). These codes (for FIFO, PQ, CQ, WFQ, CBWFQ and LLQ) can be found in APPENDIX.

\section{MEASUREMENT RESULTS}

As in the previous works [9]-[10], the length of the measurement time was 5 minutes in each case. The measurements were recorded in every second. Easy to observe in the traffic generation code, that the generated voice traffic average was 513 packets per second. As in previous articles (see e.g. [8]-[11]) the following areas were examined: packet loss, end-to-end delay and jitter (delay variation).

Concerning the packet loss of voice packets (see Fig. 4) LLQ and PQ algorithms have proven to be most effective, followed by the CBWFQ. It can be observed that while the previous works based on simulation results concluded that WFQ was the best congestion management algorithm, our measurement results showed, that the WFQ performance was behind the performance of LLQ, PQ and CBWFQ. The CQ has the next poor algorithm performance, while the least efficient queuing scheduler was the FIFO.

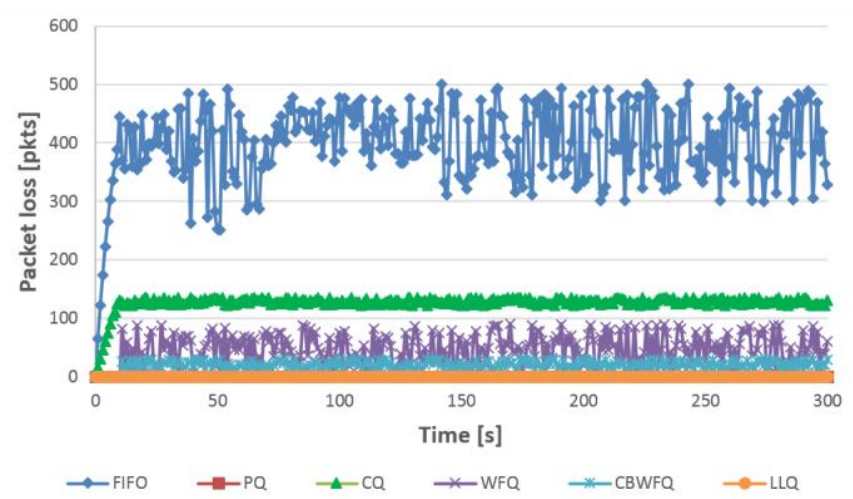

Fig. 4. VoIP packet loss

Fig. 5 shows the same content as Fig. 4, except that the former does not include the efficiency representation of the two least efficient congestion management algorithm. Thus it is prominently observable the difference of performance of $P Q$, WFQ, CBWFQ and LLQ in packet loss. Easy to see, that in the case of LLQ and PQ there was no packet loss due to the absolute priority queue, in which the real-time voice was classified. Subsequently, CBWFQ performance was the most effective, and finally the WFQ's.

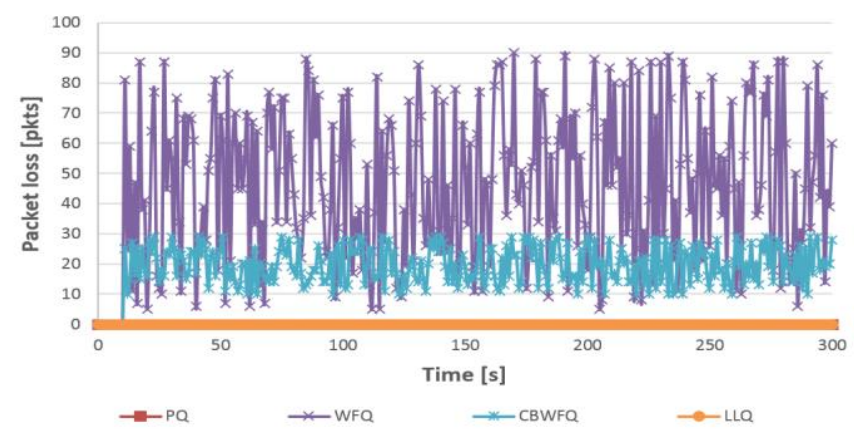

Fig. 5. VoIP packet loss for PQ, WFQ CBWFQ and LLQ

With respect of voice packet delay (see Fig. 6 and Fig. 7), LLQ and PQ algorithms managed to squeeze those values below $100 \mathrm{~ms}$, while the CQ has under $255 \mathrm{~ms}$, which already exceeds the threshold set by the QoS requirement. It is clear that in the case of WFQ and CBWFQ the delay is a little more than 1 second, while in the case of FIFO than can reach up to 8.5 seconds, which are unacceptable values provided by the QoS requirements.

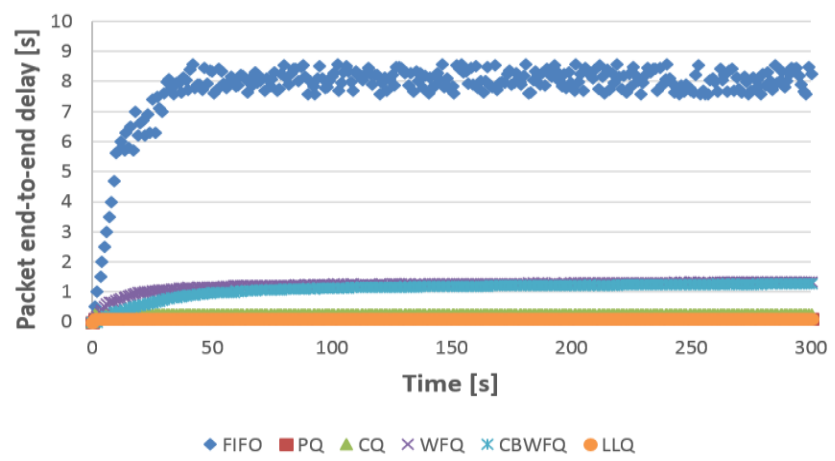

Fig. 6. VoIP traffic delay

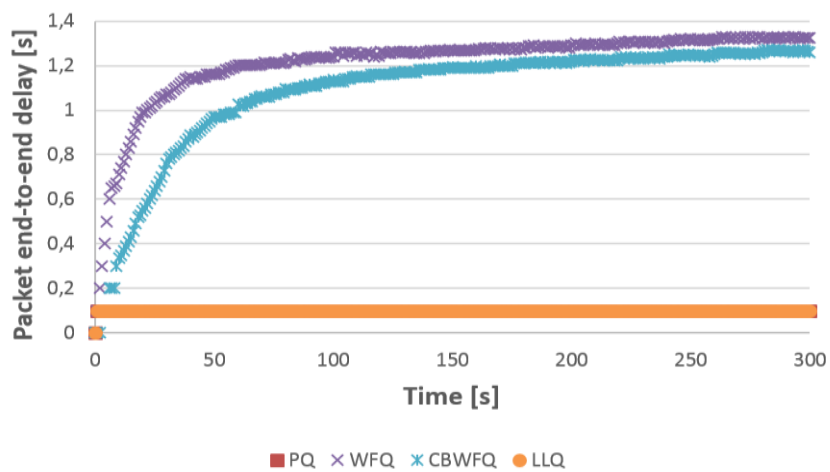

Fig. 7. VoIP traffic delay for PQ, WFQ, CBWFQ and LLQ

As for the delay variation (jitter) (see Fig. 8 and Fig. 9) the LLQ, PQ and CQ has managed to keep the measured values below 30ms. Subsequently, the WFQ and CBWFQ ensured around $150 \mathrm{~ms}$ and $210 \mathrm{~ms}$ jitter, while the FIFO has finally managed to stabilize its delay variation around 1 second. It 
should be noted that in terms of jitter PQ and LLQ congestion management algorithms managed to meet under the requirements of the QoS threshold requirement.

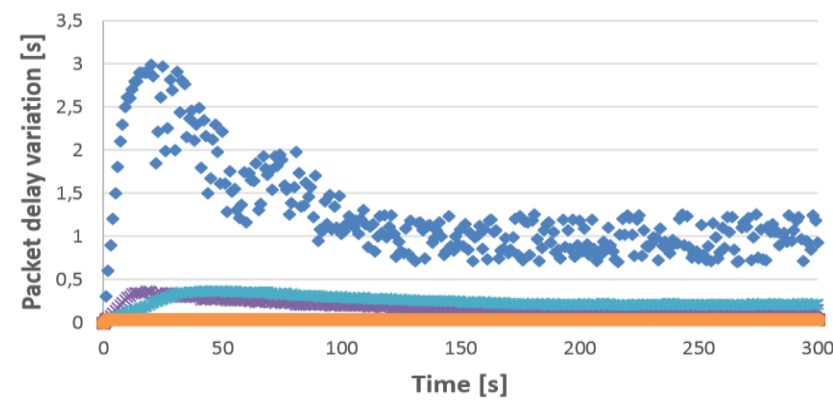

- FIFO $\triangle \mathrm{PQ} \triangle \mathrm{CQ} \times \mathrm{WFQ} * \mathrm{CBWFQ}-\mathrm{LLQ}$

Fig. 8. VoIP jitter

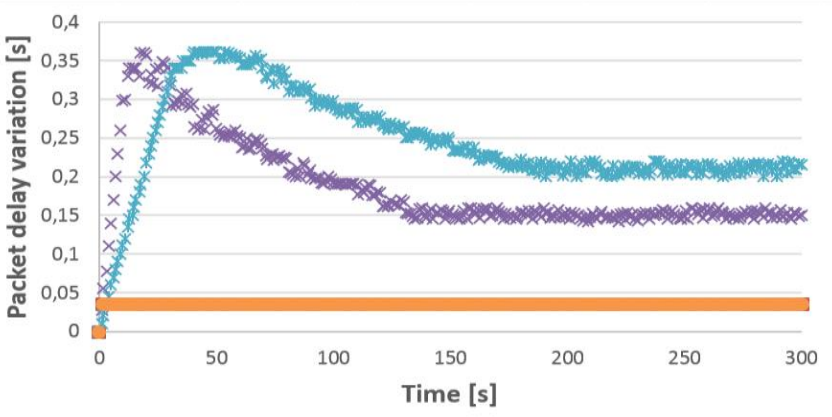

- $\mathrm{PQ} \times \mathrm{WFQ} *$ CBWFQ - LLQ

Fig. 9. VoIP jitter for PQ, WFQ, CBWFQ and LLQ

\section{CONCLUSION}

This paper compares the performance of the main congestion management algorithms based on a measurement environment. The laboratory environment was implemented at the Faculty of Informatics University of Debrecen. In all cases the measurement result shows that the FIFO scheduling principle is the most inconvenient algorithm for handling of interactive voice packets in case of congestion. In the case of voice transmission the PQ and the LLQ algorithms were the two most appropriate algorithms, in terms of packet loss rate, end-to-end delay and jitter. Using these algorithms no packets suffered packet loss. However, knowing the principle of the $\mathrm{PQ}$, namely that it serves the maximum priority queue, but produce packet starvation for other tree queues, based on the literature and on the measurement results the conclusion is, that for the interactive real-time voice traffic, taking all in consideration, the LLQ congestion management algorithm is most appropriate. Further research topic is to support the results and test the algorithms presented in the current article by mathematical modeling.

\section{APPENDIX}

These router configuration settings were used for implementing the congestion management algorithms:

\section{FIFO}

int $\mathrm{s} 0 / 1 / 1$ no fair-queue

end

$\mathbf{P Q}$

access-list 101 permit tcp 172.16.10.0 0.0.0.255

$172.16 .20 .0 \quad 0.0 .0 .255$ eq 21

access-list 102 permit tcp 172.16 .10 .00 .0 .0 .255

$172.16 .20 .0 \quad 0.0 .0 .255$ eq 1720

access-list 103 permit udp 172.16.10.0 0.0.0.255

$172.16 .20 .0 \quad 0.0 .0 .255$ eq 5060

priority-list 1 protocol ip high list 103

priority-list 1 protocol ip medium list 102

priority-list 1 protocol ip normal list 101

priority-list 1 default low

priority-list 1 queue-limit 20406080

int $\mathrm{s} 0 / 1 / 1$

priority-group 1

end

CQ

access-list 101 permit tcp 172.16.10.0 0.0.0.255

172.16 .20 .00 .0 .0 .255 eq 21

access-list 102 permit tcp 172.16.10.0 0.0.0.255

$172.16 .20 .0 \quad 0.0 .0 .255$ eq 1720

access-list 103 permit udp 172.16.10.0 0.0.0.255

$172.16 .20 .0 \quad 0.0 .0 .255$ eq 5060

queue-list 1 protocol ip 2 list 103

queue-list 1 protocol ip 3 list 102

queue-list 1 protocol ip 4 list 101

queue-list 1 default 1

queue-list 1 queue 1 limit 4

queue-list 1 queue 2 limit 10

queue-list 1 queue 3 limit 10

queue-list 1 queue 4 limit 4

int $\mathrm{s} 0 / 1 / 1$

custom-queue-list 1

end

\section{WFQ}

int s0/1/1

fair-queue

end

\section{CBWFQ}

access-list 101 permit tcp 172.16.10.0 0.0.0.255

172.16 .20 .00 .0 .0 .255 eq 21

access-list 102 permit tcp 172.16 .10 .00 .0 .0 .255

172.16 .20 .0 0.0.0.255 eq 1720

access-1ist 103 permit udp 172.16.10.0 0.0.0.255

172.16 .20 .0 0.0.0.255 eq 5060

class-map VOICE

match access-group 103

exit

class-map VIDEO

match access-group 102

exit

class-map FTP

match access-group 101

exit

policy-map R1-Serial

Class VOICE

bandwidth percent 30

exit

class VIDEO

bandwidth percent 30

exit

class FTP

bandwidth percent 10

exit

class class-default

bandwidth percent 5

exit

exit

int $\mathrm{s} 0 / 1 / 1$

no fair-queue

service-policy output R1-Serial 
end

\section{LLQ}

access-list 101 permit top $172.16 .10 .0 \quad 0.0 .0 .255$

172.16 .20 .0 0.0.0.255 eq 21

access-list 102 permit tcp $172.16 .10 .0 \quad 0.0 .0 .255$

$172.16 .20 .0 \quad 0.0 .0 .255$ eq 1720

access-list 103 permit udp $172.16 .10 .0 \quad 0.0 .0 .255$

$172.16 .20 .0 \quad 0.0 .0 .255$ eq 5060

class-map VOICE

match access-group 103

exit

class-map VIDEO

match access-group 102

exit

class-map FTP

match access-group 101

exit

policy-map R1-Serial

Class VOICE

priority 384

exit

class VIDEO

bandwidth percent 30

exit

class FTP

bandwidth percent 10

exit

class class-default

bandwidth percent 5

exit

exit

int $\mathrm{s} 0 / 1 / 1$

no fair-queue

service-policy output R1-Serial

end

\section{REFERENCES}

[1] QOS, "Implementing Cisco Quality of Service, Student Guide", Ver. 2.2, vol. 2, Cisco Systems Inc., 2006.

[2] A. S. Tanenbaum and D. J. Wetherall, "Computer Networks", 5th Edition, Pretince Hall, ISBN-13: 978-0132126953, 2013.

[3] T. Svensson and A. Popescu, "Development of laboratory exercices based on OPNET Modeler", Blekinge Institute of Technology, Department of Telecommunications and Signal Processing, 2003.

[4] C. Mancas and M. Mocanu, "QoS Optimization in Congested Multimedia Networks," Proceedings of the 36th TSP Conference, pp. 38-42, 2013.
[5] "Cisco IOS Quality of Service Solutions Configuration Guide", Release 12.4T, Cisco Systems Inc., 2008.

[6] W. Odom, J. Geier and N. Mehta, "CCIE Routing and Switching Official Exam Certification Guide", 2nd Edition, Cisco Press, 2006.

[7] A. S. Ranjbar, „CCNP ONT Official Exam Certification Guide”, Cisco Press, 2007.

[8] M. M. G. Rashed and M. Kabir, "A Comparative Study off Different Queuing Techniques in VoIP, Video Conferencing and FTP," Daffodil International University Journal of Science and Technology, vol. 5, no. $1,2010$.

[9] S. Szilágyi, "The Effects of Different Queuing Techniques over VoIP Performance: A Simulation Approach," in Abstracts \& Pre-Proceedings of the 9th International Conference on Applied Mathematics, Baia Mare, Romania, 2013.

[10] S. Szilágyi, "Analysis of the Algorithms for Congestion Management in Computer Networks," Carpathian Journal of Electronic and Computer Engineering, vol. 6, no. 1, pp. 3-7, 2013.

[11] S. Akhtar, E. Ahmed, A. k. Saka and K. S. Arefin, "Performance Analysis of Integrated Service over Differentiated Service for Next Generation Internet," JCIT, vol. 1, no. 1, 2010.

[12] S. Szilágyi and B. Almási, "A Review of Congestion Management Algorithms on Cisco Routers," Journal of Computer Science and Control Systems, vol. 5, no. 1, 2012.

[13] A. Kuki, T. Bérczes, B. Almási, J. Sztrik, "A Queing Model to Study the Effect of Network Service Breakdown in a CogInfoCom System", Cognitive Infocommunication (CogInfoCom) 2013, 4th IEEE International Conference on Cognitive Infocommunications, IEEE Publisher, pp. 205-2010, ISBN: 978-1-4799-1543-9, 2013.

[14] B. Almási, G. Bolch, D. Tutsch, "Stochastic Modeling of Multistage Interconnection Networks with MOSEL", Journal of Mathematical Sciences, vol. 121, no. 5, 2004.

[15] L. L. Peterson and B. S. Davie, "Computer Networks: A System Approach - Network Simulation Experiments Manual", 3rd Edition, Elsevier Inc., 2011.

[16] A. S. Sethi and V. Y. Hnatyshin, "The Practical User Guide for Computer Network Simulation", NW: CRC Press Taylor \& Francis Group, 2012.

[17] A. Cisco Networking, „Pagent IOS Tutorial,” Cisco Systems Inc., 2009.

[18] A. Cisco, "CCNP: Optimizing Converged Networks v5.0 - Instructor Lab Manual," Cisco Systems, Inc., 2007.

[19] C. Cisco, „TGN (Traffic GeNerator) User Manual,” Cisco Systems Inc., 2007. 